\title{
BAYESIAN METHODS: WHAT CAN WE GAIN AND AT WHAT COST?
}

Much has been written about the use of Bayesian methods in archaeology, and in particular in the analysis and interpretation of radiocarbon dates. The idea is deceptively simple and has been "around" for over 200 years. However, Bayesian methods have not been widely used in practical problems till the last decade, due to the computational difficulties which they may present.

The deceptively simple idea is that we can take into account prior information or beliefs when interpreting the evidence, in the case discussed in this issue's P Steier and W Rom paper, this involves incorporating information about stratigraphy into the analysis of the ${ }^{14} \mathrm{C}$ dates. It requires an expression of the prior information in a probabilistic manner and some quite sophisticated software. Knowledge and understanding of the methods are required to ensure that any assumptions are appropriate.

The immediate difficulty, which cannot be over-emphasized, is in the specification of the prior information. There are however, prior descriptions which are commonly described as uninformative and which in principle make no strong assumption, and which are the default options described in the paper by Steier and Rom and available in OxCal. In practice, the assumption of an "uninformative" prior should mean that the data determines the final outcome. They show that this "uninformative" prior in fact makes strong assumptions and has considerable influence on the final results. However, one solution to the problem does exist and is available within OxCal, namely the bounding option.

What can potentially be gained by the use of Bayesian methods? Well, in principle, use of Bayesian methods means that many different types of information can be taken into account in the analysis, which should therefore result in that analysis becoming more precise (more information should equate to better precision). In many archaeological and other problems, increased precision is an important goal, allowing us to identify smaller, real differences. The paper has however shown that Bayesian analysis is not a "cure-all"; it has costs, not least the specification of the prior. This is not easy and even in those situations where we think we are not making any strong assumptions, there may be hidden complications, as their paper has shown.

I support the final comment of the two authors that specification of the prior needs to be made with care and in collaboration with a variety of specialists, not simply the archaeologists.

\section{Marian Scott}

